\title{
How to write a manuscript for Sports Engineering
}

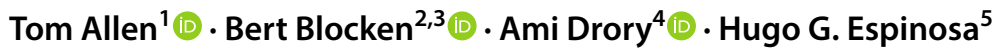

Accepted: 17 October 2020 / Published online: 20 November 2020

(c) International Sports Engineering Association 2020

This editorial provides guidance for authors preparing a manuscript for submission to Sports Engineering. It does not replace the submission guidelines on the journal webpage, ${ }^{1}$ nor does it simply duplicate the scientific paper writing guides that are already available [1-8]. The submission guidelines on the journal webpage provide specific instructions that authors must adhere to, including details on article types and associated word and figure/table limits, how the manuscript should be formatted and structured and referencing style. The submission guidelines are periodically reviewed and updated as required.

This editorial focuses on scientific issues, offering guidance that is specific to Sports Engineering, based on our observations as editors to make it easier for authors to submit clear research reports that are more likely to be published. The two manuscript types published in Sports Engineering, besides review articles that are typically invited, are original research articles and technical notes. Indeed, work presenting a new method, test rig or measurement device should be submitted as a technical note. Authors should not think of technical notes as being lesser than research articles, rather they should simply think of them as another type of publication, which is particularly relevant to the field of sports engineering and technology. This editorial is structured around the main sections of a typical manuscript, including

Tom Allen

t.allen@mmu.ac.uk

1 Department of Engineering, Manchester Metropolitan University, Manchester, United Kingdom

2 Department of the Built Environment, Eindhoven University of Technology, Eindhoven, Netherlands

3 Department of Civil Engineering, KU Leuven, Leuven, Belgium

4 Faculty of Engineering, University of Nottingham, Nottigham, United Kingdom

5 School of Engineering and Built Environment, Griffith University, Brisbane, Queensland, Australia the Title, Abstract, Introduction, Methods, Results, Discussion and Conclusion.

A good Title is vital, and it should be concise and clear, such as in the form of "Effect of A on B" for research articles, e.g. "Effect of surrogate design on the measured stiffness of snowboarding wrist protectors" [9]. Words used in the title should be specific (precise) rather than general (vague) and jargon should be avoided. It is sometimes advantageous to indicate the main finding in the title. A title for a technical note should clearly state the intended purpose of the new method, test rig or measurement device, e.g. "Measuring temporal and spatial accuracy in trampolining" [10]. A good title includes key words describing the topic or methods such that it is more likely to be found when searching online.

The Abstract should provide a concise summary of the manuscript, clearly summarising the introduction, method, results, discussion and conclusions [11]. The rationale for the work should be clearly stated, as should the key finding or 'message'. In most instances, the key findings should be quantified. Do not include references, abbreviations/ acronyms and brand names; indeed the use of the latter two should always be kept to a minimum throughout a manuscript. Try to finish on a strong 'take-home message', but avoid overstating or overextending your findings.

The Introduction should summarise and synthesise the results of previous original research on the topic. When describing previous research, authors should define lesser known terms and use them consistently throughout the manuscript to avoid confusion and misconception $[12,13]$. Standard International System of Units (SI) definitions must also be adhered to throughout the manuscript [14]. Attention should be paid to the level of evidence of previous research in weighing past results and the evidence needed for application $[15,16]$. Without a clear rationale and justification for the research, it is hard to justify publication. Indeed, the reported research must provide some meaningful, novel contribution to sports engineering knowledge or application.

$\overline{1}$ https://www.springer.com/journal/12283/submission-guidelines. 

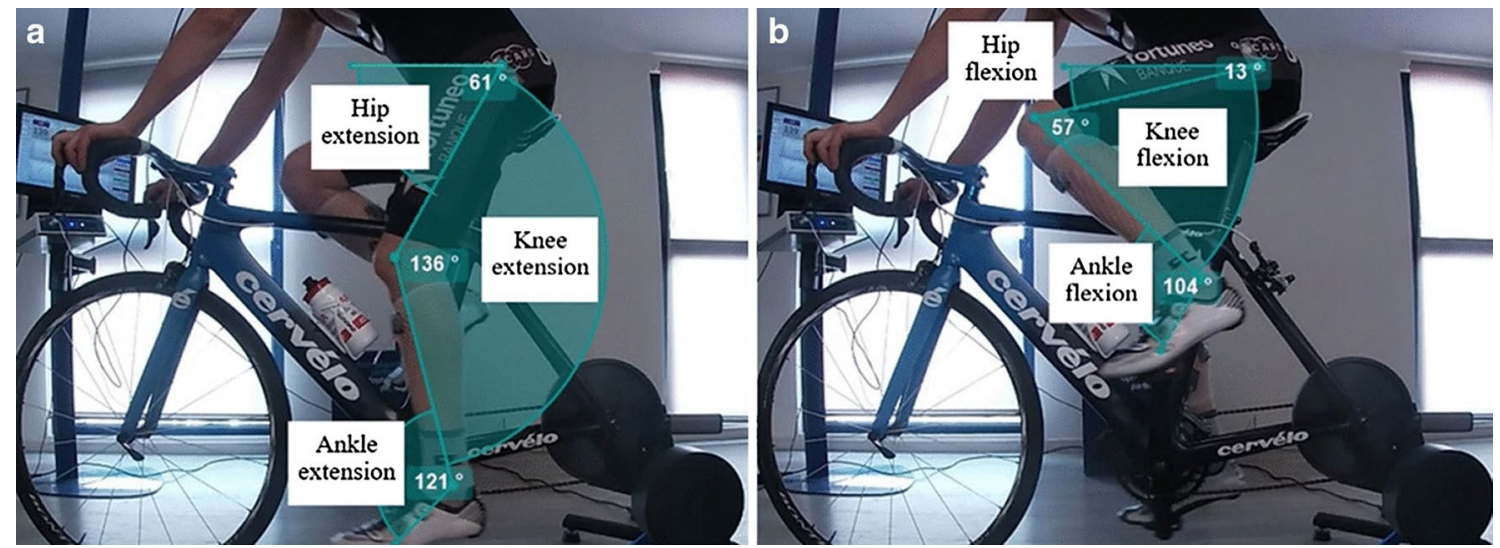

Fig. 1 Measurements of the hip, knee and ankle angles (degrees) during the extension (a) and the flexion (b) of the left lower limb while pedalling (GoPro images displayed in Kinovea software) [17]

Fig. 2 Diagrams showing a the measured geometric and mass properties and $\mathbf{b}$ moments of inertia of a tennis racket. c Methods for measuring handle length of old wooden rackets. Dates are: top 1916, middle 1911, bottom 1877 [18]

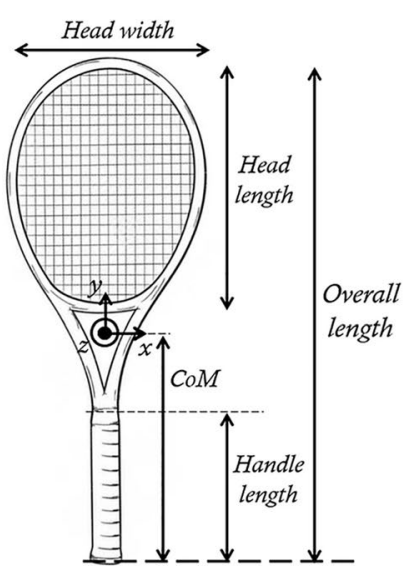

a
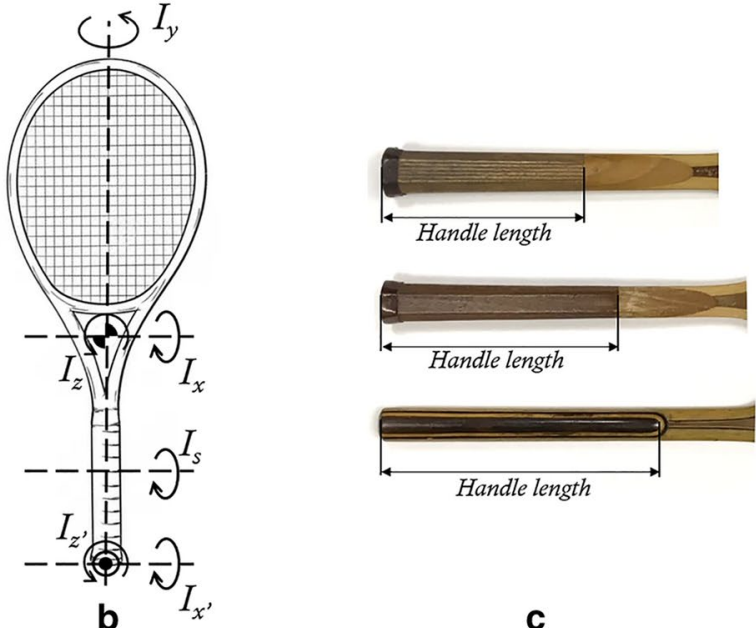

Handle length

C
The Methods section is often the key for Sports Engineering articles, and must be presented so that others can reproduce the work. Reproducibility is a prerequisite for acceptance by the scientific community. As such, detailed and clear explanations of methods, including any equipment used, should be included. Clearly annotated figures (e.g. Fig. 1; [17]) can help communicate methods to the reader. Subplots and multi-panel figures (e.g. Fig. 2; [18]) can be used to efficiently summarise important information, such as operational definitions of variables, with the added benefit of minimising the total number of figures. Authors are encouraged to use online supplementary materials for text, figures, data and videos (e.g. $[19,20])$ that communicate important information. Such supplementary material can help to support a more 'open' scientific culture [21].

When introducing equipment and measurement devices, such as wearable sensors, details of the make and model should be stated. As research articles must be objective, generic descriptions, rather than brand names, must be used to refer to the device throughout the manuscript. It is also important to state how such devices were calibrated, and synchronised if more than one was used, and for wearable sensors their specific location on the body or equipment $[22,23]$. An estimation of measurement error should also be provided for measurement devices [8, 24], which can come from the authors own testing, published work or manufacturer specifications. When benchmarking a device, or model, against a reference standard, it is better to quantify differences (validity) and variability (reliability), rather than simply using words like 'validated' or 'good agreement'. When reporting results, the number of significant figures should correspond with the reported measurement accuracy, to avoid implying unrealistically high precision [12]. As such, the number of significant figures by which results are reported must be justified. For example, when measuring the size of tennis rackets (Fig. 2), Taraborrelli and colleagues [18] stated that they used a tape measure with a resolution of $1 \mathrm{~mm}$, and then 
Table 1 Geometric properties of 416 tennis rackets, dating from 1874 to 2017 , as defined in Fig. 2 [18]

\begin{tabular}{lllllll}
\hline Overall length $(\mathrm{m})$ & Handle length $(\mathrm{m})$ & \multicolumn{2}{l}{ Head length $(\mathrm{m})$} & & \multicolumn{2}{l}{ Head width $(\mathrm{m})$} \\
\cline { 3 - 4 } & & External & Internal & & External & Internal \\
\hline $0.686 \pm 0.010$ & $0.196 \pm 0.036$ & $0.315 \pm 0.032$ & $0.292 \pm 0.031$ & & $0.234 \pm 0.021$ & $0.209 \pm 0.021$ \\
$0.652-0.811$ & $0.148-0.355$ & $0.240-0.493$ & $0.226-0.455$ & & $0.185-0.308$ & $0.157-0.285$ \\
\hline
\end{tabular}

Mean \pm standard deviation, range appropriately reported descriptive statistics to the nearest millimeter (Table 1).

When the method involves computational or mathematical approaches, the authors should precisely describe the approaches used, while justifying simplifications and assumptions. For example, machine learning is a broad field incorporating many techniques, so it is not helpful to just state that one used machine learning techniques. Articles presenting results obtained by numerical simulation with computational fluid dynamics (CFD) should report the grid topology, should include a validation study by comparison with experimental data and should have been obtained with at least second-order discretization schemes. Best practice guidelines in CFD such as those by ERCOFTAC [25] should be adhered to. Similar guidelines should be adhered to when using the finite element method (FEM) for structural analysis of sports equipment, such as those from the National Agency for Finite Element Methods and Standards (NAFEMS) [26] on a range of topics covering engineering simulation.

When referring to wearable sensors, it is more useful to provide details or features of the main parts rather than just writing a "bespoke inertial measurement unit (IMU)", or using a brand name. For bespoke measurement devices, authors should state the make and model of the first principles sensors. For example, a bespoke IMU should state the make and model of the accelerometer, gyroscope and magnetometer, as well as the corresponding measurement ranges and sampling frequency [e.g. 23, 27]. Likewise, bespoke software or script should detail the algorithms and functions implemented or used. Similar guidance applies when testing sports equipment, like running shoes, tennis rackets or helmets. Rather than just stating the make and model of sports equipment, it is better to also compare details of the main parts and design parameters, noting dimensions, materials and other basic properties. Including such detail offers more opportunity for furthering reader understanding when they are not familiar with the specific equipment. Omitting such details of equipment and devices is not helpful to our readers, and makes it hard to justify publication, particularly if it prevents the work from being reproducible. Again, as research articles must be objective, authors should cover up brand names and logos on images of sports equipment, and avoid using such identifiers in latter sections, including on figures. If possible, it is generally best to test materials and equipment against relevant standards, with justification needed for alternative approaches.

If the work involves testing with humans, animals or biological material, then prior approval is required from an ethics committee. ${ }^{2}$ There should be a statement in the body of the text confirming that ethical approval was granted, and the work was performed in accordance with the Declaration of Helsinki. These ethical requirements still apply if an author was the sole participant, such as when collecting data to benchmark the accuracy of a device or sensor, to assess sports equipment or as an input for a model. There are exceptions where obtaining prior ethics approval may not be possible, such as a retrospective study using data collected on professional athletes as part of their job, but the source of the data and the reason for the lack of prior ethics approval must be stated [28]. Participants should be referred to as such or other descriptive term (e.g., athletes, patients), not "subjects", with a statement confirming that they gave free and informed consent, in writing.

As with measurement devices, sports equipment and models, key details of any participants, such as their age, mass, height and sex, should be included. Such information should be presented unambiguously, so instead of stating "the average age of the participants was $32 \pm 3$ years", it is better to write "the mean age of the participants was $32 \pm 3$ years, where \pm refers to the standard deviation", since “ \pm " can refer to standard deviation or standard error [29], or even perhaps something else entirely. The inclusion and exclusion criteria for the study and the exact sample size should also be stated. The sample size should be justified, and depending on the nature of the research this may mean using an a priori power analysis given the size of effect sought, variability expected, and appropriate statistical error rates [30-34]. Indeed, appropriate statistical analysis is needed to see if an intervention, such as a change to the mass of a running shoe, has an influence on athlete performance. Authors should clearly report all data and justify the use of inferential statistics, and size of effect statistics. A detailed discussion on the use of statistics falls outside the scope of this editorial, and authors seeking further guidance are referred to the literature $[8,30-37]$.

\footnotetext{
2 https://www.springer.com/gp/editorial-policies/research-involvinghuman-and-or-animal-participants.
} 


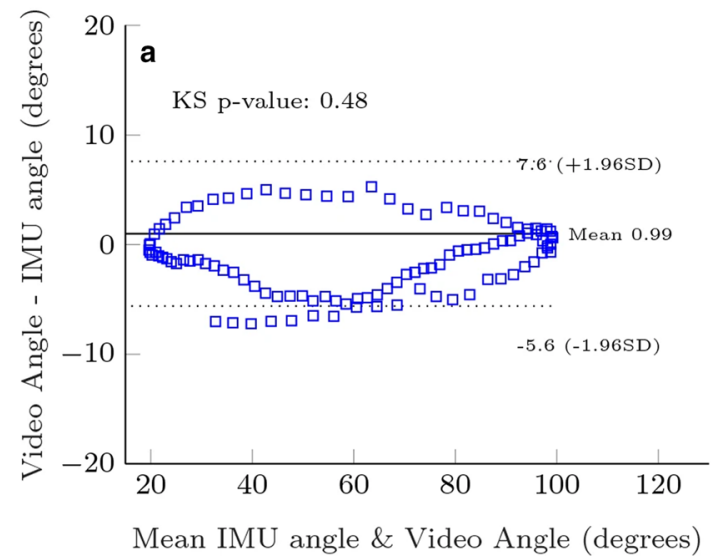

Fig. 3 Sample Bland-Altman and correlation analysis. a Bland-Altman plot compares the pole lean angles estimated by the IMU against those measured by video. Solid line illustrates the bias of the mean

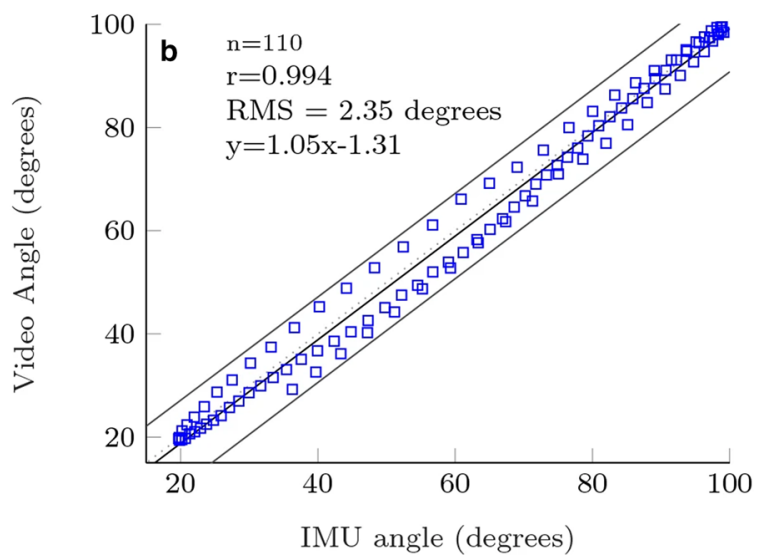

differences $(\bar{d}=0.99)$ and the dotted lines illustrate the $95 \%$ CI of the agreement limits $(-5.6,7.6)$. b Correlation plot with the superimposed best-fit line (solid) and 95\% CI (dashed) [27]

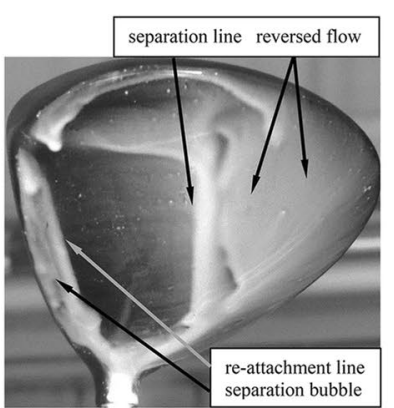

a $\mathrm{U}=26 \mathrm{~m} / \mathrm{s}$ no DDE (clean)

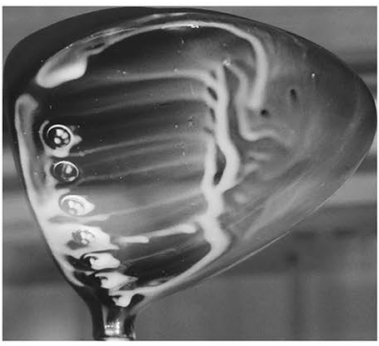

b $U=26 \mathrm{~m} / \mathrm{s} x=15 \mathrm{~mm}, \mathrm{~d}=7 \mathrm{~mm} \mathrm{~s}=7 \mathrm{~mm}$

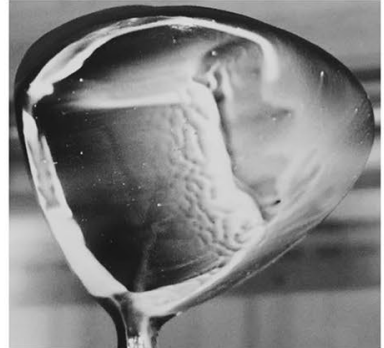

$\mathrm{U}=36 \mathrm{~m} / \mathrm{s}$ no DDE (clean)

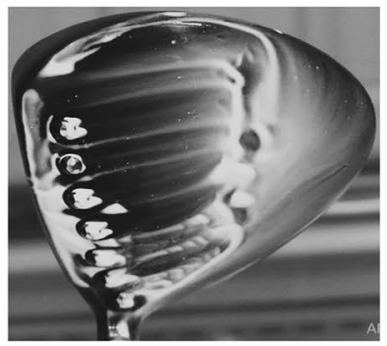

$\mathrm{U}=36 \mathrm{~m} / \mathrm{s} \mathrm{x}=15 \mathrm{~mm}, \mathrm{~d}=7 \mathrm{~mm} \mathrm{~s}=7 \mathrm{~mm}$

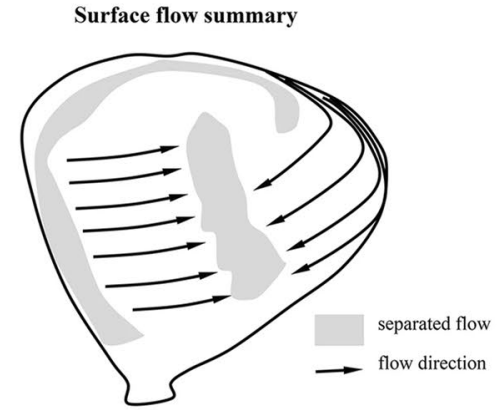

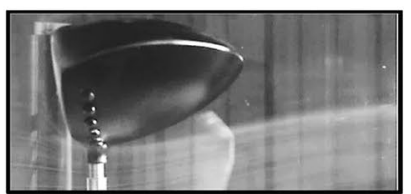

(c) $U=26 \mathrm{~m} / \mathrm{s} x=15 \mathrm{~mm}, \mathrm{~d}=7 \mathrm{~mm} \mathrm{~s}=7 \mathrm{~mm}$

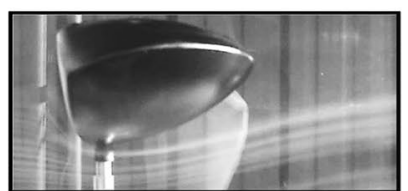

no DDE

Fig. 4 On-surface skin friction patterns over a the clean club and b with DDE attachment and off-surface smoke visualisation over the $\mathbf{c}$ $x=15 \mathrm{~mm}, d=7 \mathrm{~mm}, \mathrm{~s}=7 \mathrm{~mm}$ DDEs and clean club at $U=26 \mathrm{~m} / \mathrm{s}$. Freestream is left to right. Sketch shows dominant flow directions [39]

The Results section should be concise, focusing on objectively presenting the data. Depending on the nature of the research, the data presented could be based on statistical tests. Data can be presented in the narrative, and either a table (e.g. Table 1) or figure, not both. Tables containing all the data can be included in the supplementary material if appropriate. A good choice of figure is vital for effectively communicating your results without bias [21]. Bland-Altman [38] and scatter plots can, for example, work well when describing the agreement between two measurements (Fig. 3; [27]). Many other types of images can be used to effectively communicate findings and concepts, such as smoke visualisations from wind tunnels (Fig. 4; [39]), and contour plots from particle image velocimetry (Fig. 5; [40]) and computer simulations (Fig. 6; [41]).

Key considerations for figures include:

- Using appropriate resolutions, font size and axis ranges, 


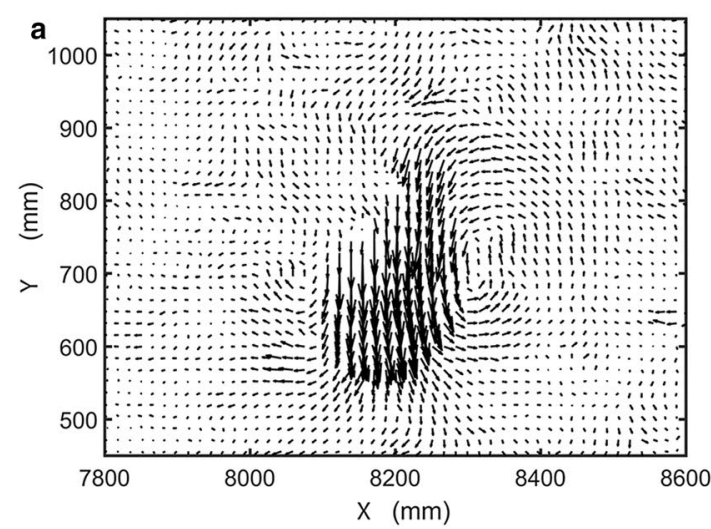

Fig. 5 The velocity field (vector plot) and the vorticity component perpendicular to the plane of visualisation (color map), in, respectively, (a) and (b), of a typical coherent vortical structure found in the

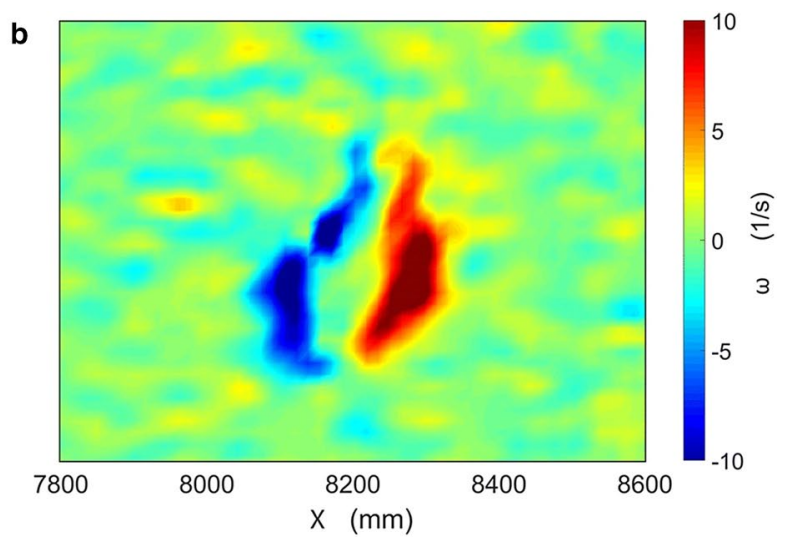

kick trials. From the vector field, the mean rising speed of the bubbles is subtracted [40]
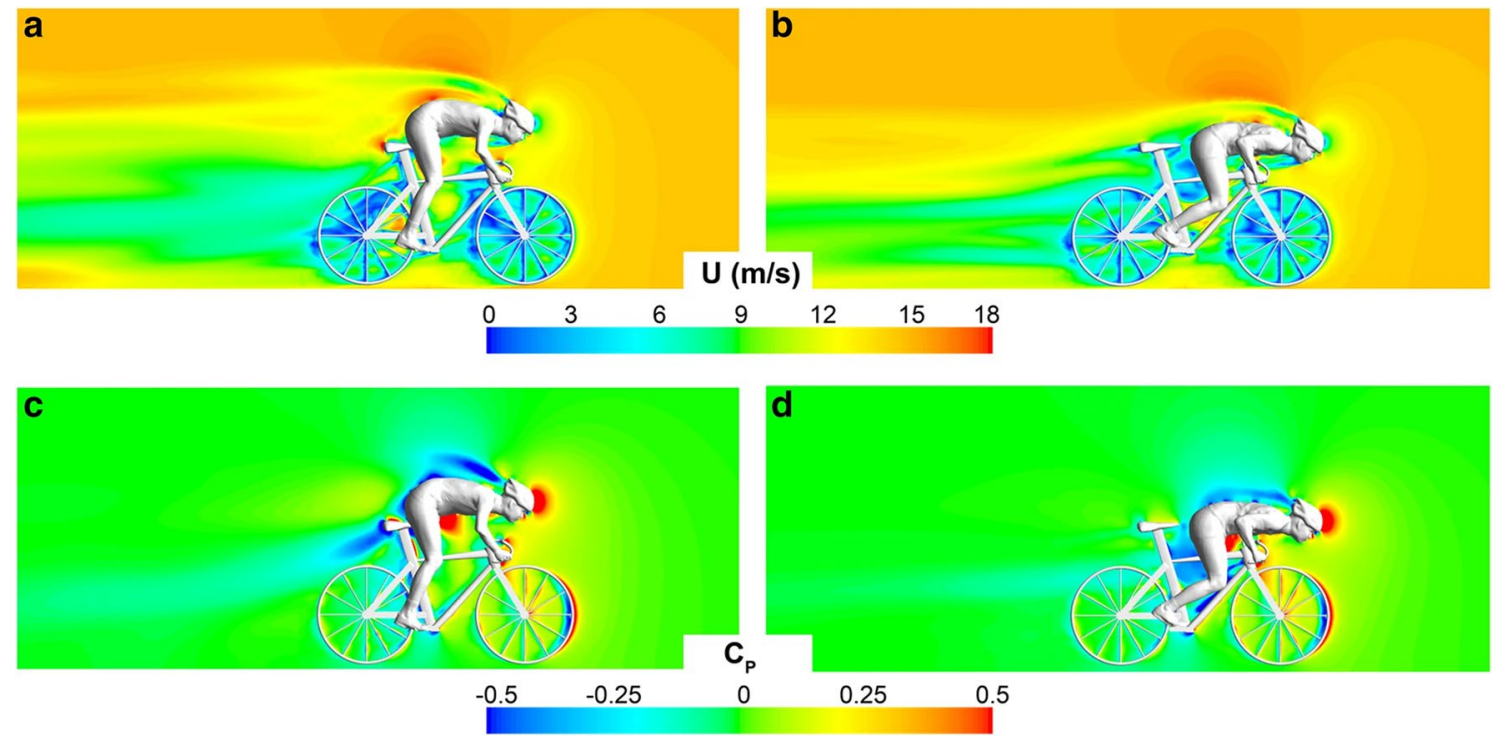

Fig. 6 Contours of $\mathbf{a}, \mathbf{b}$ mean air speed and $\mathbf{c}, \mathbf{d}$ mean static pressure coefficient in a vertical center plane for the two sprint positions [41]

- Using a standard font such as Times New Roman, Arial, or Calibri that is easy to read and will be in keeping with that of the main body of text in the published article,

- Ensuring consistency in line and marker styles, legends and other labels between plots,

- Using plotted line patterns that are distinguishable in black and white,

- Including coordinate system images to clarify directions (Fig. 2), and

- Using clear and descriptive captions.

As per the examples included here, plots should be combined into multi-panel figures where possible.
The Discussion should describe the meaning of the results, with reference to the literature and knowledge gap identified in the introduction. Avoid repetition, like restating the aim, rationale, or hypothesis of the work. Indeed, repetition should be avoided throughout the manuscript, with the exception of the abstract and conclusion. The important limitations of the work should be clearly stated and their potential influence noted. Opportunities for further work can also be noted alongside the limitations. It is often beneficial to place your data and findings within a wider context of sports engineering and technology, especially outside of the more-limited application or sporting example demonstrated in the manuscript, but again avoid overstating the findings. 
The Conclusion should clearly state the key findings, and provide a take-home message. Again, the findings should not be overstated or overextended. This is not the place to introduce new topics or references, nor cite figures.

We hope these guidelines help authors in preparing their manuscripts.

Acknowledgements We thank the reviewers who provided helpful feedback on this editorial.

\section{References}

1. Day RA, Gastel B (2017) How to write and publish a scientific paper. Cambridge University Press, Cambridge

2. Plaxco KW (2010) The art of writing science. Protein Sci Publ Protein Soc 19(12):2261

3. Buenz EJ (2019) Essential elements for high-impact scientific writing. Nature. https://doi.org/10.1038/d41586-019-00546-7

4. Bartlett R (2001) Writing for the Journal of Sports Sciences. J Sports Sci 19(7):467

5. Winter E (2005) Writing: Bartlett revisited. J Sports Sci 23(8):773. https://doi.org/10.1080/02640410500217156

6. Blocken B (2017) 10 Tips for writing a truly terrible journal article. Elsevier Connect, Author's Update

7. Lipton ZC (2018) Heuristics for scientific writing (a machine learning perspective) https://approximatelycorrect .com/2018/01/29/heuristics-technical-scientific-writing-machi ne-learning-perspective/. Accessed 6 Aug 2020

8. Knudson D (2009) Significant and meaningful effects in sports biomechanics research. Sports Biomech 8(1):96-104

9. Adams C, James D, Senior T, Allen T, Hamilton N (2018) Effect of surrogate design on the measured stiffness of snowboarding wrist protectors. Sports Eng 21(3):217-225

10. Ferger K, Hackbarth M, Mylo MD, Müller C, Zentgraf K (2019) Measuring temporal and spatial accuracy in trampolining. Sports Eng 22(3-4): 18

11. How to construct a Nature summary paragraph, annotated example taken from Nature 435:114-118 (5 May 2005). https://natur e.com/documents/nature-summary-paragraph.pdf. Accessed 6 Aug 2020

12. Winter EM (2008) Use and misuse of the term "significant." J Sports Sci 26:429-430

13. Vigotsky AD, Zelik KE, Lake J, Hinrichs RN (2019) Mechanical misconceptions: have we lost the "mechanics" in "sports biomechanics"? J Biomech 93:1-5

14. BIdPe M (2014) SI brochure: the international system of units (SI). International Bureau of Weights and Measures. https://www. bipm.Org2014. Accessed 8 Oct 2020

15. Knudson D, Elliott B, Hamill J (2014) Proposing application of results in sport and exercise research reports. Sports Biomech 13(3):195-203

16. Knudson D, Elliott B, Ackland T (2012) Citation of evidence for research and application in kinesiology. Kinesiol Rev 1(2):129-136

17. Millour G, Duc S, Puel F, Bertucci W (2020) Effect of asymmetric crank arm lengths on performance-related variables in cyclists with an anatomical lower limb length discrepancy. Sports Eng 23(1):1-9

18. Taraborrelli L, Grant R, Sullivan M, Choppin S, Spurr J, Haake $S$, Allen T (2019) Recommendations for estimating the moments of inertia of a tennis racket. Sports Eng 22(1):11
19. Blocken B, Malizia F, van Druenen T, Gillmeier S (2020) Aerodynamic benefits for a cyclist by drafting behind a motorcycle. Sports Eng 23(1):1-11

20. Hermann A, Ostarhild J, Mirabito Y, Bauer N, Senner V (2020) Stretchable piezoresistive vs. capacitive silicon sensors integrated into ski base layer pants for measuring the knee flexion angle. Sports Eng. https://doi.org/10.1007/s12283-020-00336-9

21. Halperin I, Vigotsky AD, Foster C, Pyne DB (2018) Strengthening the practice of exercise and sport-science research. IJSPP 13(2). https://journals.humankinetics.com/view/journals/ijspp /13/2/article-p127.xml

22. Mally F, Hofstätter O, Eckelt M (2020) In-shoe plantar pressure measurement-influence of insole placement on selected parameters during running. In: Multidisciplinary digital publishing institute proceedings, vol 49, no 1, p 50

23. Worsey MT, Espinosa HG, Shepherd JB, Lewerenz J, Klodzinski FS, Thiel DV (2020) Features observed using multiple inertial sensors for running track and hard-soft sand running: a comparison study. . In: Multidisciplinary digital publishing institute proceedings, vol 49, no 1 , p 12

24. Krumm D, Schwanitz S, Odenwald S (2020) How to assess repeatability and reproducibility of a mechanical test? An example for sports engineers. . In: Multidisciplinary digital publishing institute proceedings, vol 49, no $1, \mathrm{p} 122$

25. Casey M, Wintergerste T (2000) Best practice guidelines, ERCOFTAC special interest group on quality and trust in industrial CFD, ERCOFTAC, Brussels

26. NAFEMS https://www.nafems.org/. Accessed 6 Aug 2020

27. Bruzzo J, Perkins NC, Mikkola A (2020) Embedded inertial measurement unit reveals pole lean angle for cross-country skiing. Sports Eng 23(1):1-10

28. Winter EM, Maughan RJ (2009) Requirements for ethics approvals. J Sports Sci 27(10):985

29. Altman DG, Bland JM (2005) Standard deviations and standard errors. BMJ 331(7521):903

30. Sullivan GM, Feinn R (2012) Using effect size-or why the P value is not enough. $\mathrm{J}$ Grad Med Educ 4(3):279-282

31. Harrison AJ, McErlain-Naylor SA, Bradshaw EJ, Dai B, Nunome H, Hughes GT, Kong PW, Vanwanseele B, VilasBoas JP, Fong DT (2020) Recommendations for statistical analysis involving null hypothesis significance testing. Sports Biomech. https://www.tandfonline.com/doi/full/10.1080/14763 141.2020.1782555

32. Perezgonzalez JD (2015) Fisher, Neyman-Pearson or NHST? A tutorial for teaching data testing. Front Psych 6:223

33. Lakens D (2013) Calculating and reporting effect sizes to facilitate cumulative science: a practical primer for t-tests and ANOVAs. Front Psychol 4:863

34. Knudson D (2017) Confidence crisis of results in biomechanics research. Sports Biomech 16(4):425-433

35. Winter EM, Abt GA, Nevill AM (2014) Metrics of meaningfulness as opposed to sleights of significance. J Sports Sci 32(10):901-902

36. Wilkinson M, Winter EM (2019) Estimation versus falsification approaches in sport and exercise science. J Sports Sci 37(1):3-4

37. Amrhein V, Greenland S, McShane B (2019) Scientists rise up against statistical significance. 305-307. https://www.natur e.com/articles/d41586-019-00857-9?fbclid=IwAR3 K6Pys Q9FY4togs39BSciW3YsK-Pf6EE0I19R8zxkW4GvrGBHFuz $8 \mathrm{yF} 5 \mathrm{c}$

38. Bland JM, Altman D (1986) Statistical methods for assessing agreement between two methods of clinical measurement. Lancet 327:307-310

39. Traub LW, Munson A, McBurney J (2019) Experimental study of drag reduction on a golf driver club using discrete surface elements. Sports Eng 22(2):12 
40. van Houwelingen J, Kunnen RP, van de Water W, Holten AP, van Heijst GF, Clercx HJ (2019) Flow visualisation in swimming practice using small air bubbles. Sports Eng 22(2):13

41. Blocken B, van Druenen T, Toparlar Y, Andrianne T (2019) CFD analysis of an exceptional cyclist sprint position. Sports Eng 22(1):10
Publisher's Note Springer Nature remains neutral with regard to jurisdictional claims in published maps and institutional affiliations. 